\title{
Automatic detection of artifacts in EEG by combining deep learning and histogram contour processing
}

\author{
Nooshin Bahador, Kristo Erikson, Jouko Laurila, Juha Koskenkari, Tero Ala-Kokko, Jukka \\ Kortelainen
}

\begin{abstract}
This paper introduces a simple approach combining deep learning and histogram contour processing for automatic detection of various types of artifact contaminating the raw electroencephalogram (EEG). The proposed method considers both spatial and temporal information of raw EEG, without additional need for reference signals like ECG or EOG. The proposed method was evaluated with data including 785 EEG sequences contaminated by artifacts and 785 artifact-free EEG sequences collected from 15 intensive care patients. The obtained results showed an overall accuracy of 0.98 , representing high reliability of proposed technique in detecting different types of artifacts and being comparable or outperforming the approaches proposed earlier in the literature.
\end{abstract}

\section{INTRODUCTION}

Electroencephalogram (EEG) represents a recording of the electrical activity of the brain mainly originating from the cortex. Both physiological and non-physiological artifacts are seen in the EEG even though they are not produced by the brain. These artifacts highly affect the interpretation of the signal and should be removed prior to analysis. The artifacts are especially present in the recordings of critically ill patients as these often are longer in duration and the artifacts arise from various sources including electromechanical equipment as well as staff and patient movements [1-4].

In literature, a wide range of methods have been proposed to identify artifacts in raw EEG utilizing both time and frequency domains. Most of the existing approaches rely not only on additional reference signals but also on extracting specific features [5-11].

In recent years, machine learning methods have been increasingly used for discriminating artifact-free EEG sequences from contaminated ones [12-17]. So far, there are only few methods in literature that address a fully automatic removal approach using deep learning on EEG data [18-20]. These studies focus on detecting specific types of artifacts which makes them hard to be generalized to cover artifacts resulting from different sources. Furthermore, these studies used recordings made with a full EEG cap which is challenging in the intensive care environment where electrode attachment with minimum preparation is beneficial.

Orion Pharma is gratefully acknowledged for the unrestricted financial support of the study.

N. Bahador is with the Physiological Signal Analysis Team, Center for Machine Vision and Signal Analysis, MRC Oulu, University of Oulu, Oulu, Finland (E-mail: nooshin.bahador@oulu.fi).

K. Erikson, J. Laurila, J. Koskenkari and T. Ala-Kokko are with the Research Group of Surgery, Anaesthesiology and Intensive Care, Medical
In this study, we introduce a simple yet effective method based on combination of deep learning and histogram contour processing which not only needs no auxiliary reference signal, but also can detect different types of artifact with different characteristics in both amplitude and frequency. At the same time, spatial correlation is also considered in this method, as it is applicable the recordings of multiple channels. Moreover, our aim was to train and validate a simple deep learning-based artifact removal method and explore its performance considering only forehead EEG data which has been proved to be effective in intensive care units (ICUs) where securely maintaining electrodes' contact and providing long-term highquality signal acquisition are essential [21].

\section{DATA COLLECTION}

The EEG data was collected from 15 patients treated in the ICU of Oulu University Hospital. The patients did not have a history of significant neurological disease that could have been considered to affect EEG and were 18-85 years in age. During the recording, the patients were not mechanically ventilated and were recently diagnosed with hyperactive delirium. Delirium was treated with an administration of dexmedetomidine following the ICUs standard protocol to keep the patients moderately sedated. The study was approved by local ethics committee. Written informed consent was obtained either from the patient or his/her relative.

The EEG recordings were carried out with a BrainStatus self-adhesive electrode (Bittium, Oulu, Finland) including ten EEG channels (Figure 1) and BrainStatus wireless device with a sampling frequency of $250 \mathrm{~Hz}$. From the recordings, 157030 -s sequences were extracted offline. Half of these contained artifacts and half were artifact-free. The annotation of artifacts was manually made using visual inspection.

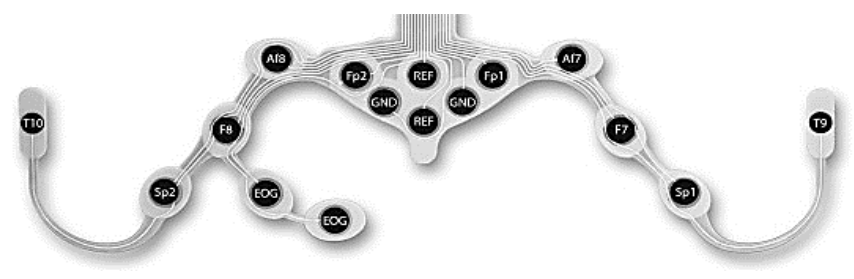

Figure 1. EEG electrode used for data collection [22]

Faculty, University of Oulu, Oulu, Finland and with the Division of Intensive Care Medicine, MRC Oulu, University of Oulu and Oulu University Hospital, Oulu, Finland.

J. Kortelainen is with the Physiological Signal Analysis Team, Center for Machine Vision and Signal Analysis, MRC Oulu, University of Oulu, Oulu, Finland and with the Cerenion Oy, Oulu, Finland. 


\section{METHOD}

Figure 2 shows the signal processing steps performed on the raw EEG data for the classification of an EEG sequence to either contain artifact or being artifact-free. First, a histogram of the EEG sequence was computed for each channel with bin number equal to the number of channels. Then, a histogram feature matrix was formed so that each row of the matrix corresponds to one channel. Next, a filled contour plot containing the isolines of the histogram feature matrix was generated (Figure 3 and Figure 4) and fed to a deep network as input image. This contour plot is an extremely simple yet useful way of summarizing and representing what simultaneously occurs in all EEG channels.

The deep learning architecture used in this model for image-to-label classification consisted of a two-dimensional convolution layer, following by a batch normalization, Relu and fully connected layers. As the contour plot is not a complex image with wide range of colors, only one convolutional layer was considered to learn contour images. The 2-D convolutional layer applied sliding convolutional filters to the input contour image. The output of this network is a categorical response and therefore a Softmax and classification layers was also added as final layers. All layers were connected sequentially. This deep network classifies 328-by-438 RGB images into the two classes i.e. artifact-free sequence and artifact-affected sequence.

The training parameters of deep learning model are given in Table. 1. The mini-batch size and the maximum number of epochs were respectively set to 64 and 8 . These small minibatches with short sequences were used to make it more suitable for training on the CPU. 5-fold cross-validation was also used to check the performance of model.

TABLE I. THE MODEL TRAINING PARAMETERS

\begin{tabular}{|c|c|c|c|}
\hline Parameter & Value & Parameter & Value \\
\hline Momentum & 0.9 & Mini Batch Size & 64 \\
\hline Initial Learn Rate & $1.0000 \mathrm{e}-03$ & Learn Rate Drop Factor & 0.05 \\
\hline L $^{2}$ Regularization & $1.0000 \mathrm{e}-04$ & Learn Rate Drop Period & 5 \\
\hline Max Epochs & 8 & Learn Rate Schedule & Piecewise \\
\hline
\end{tabular}

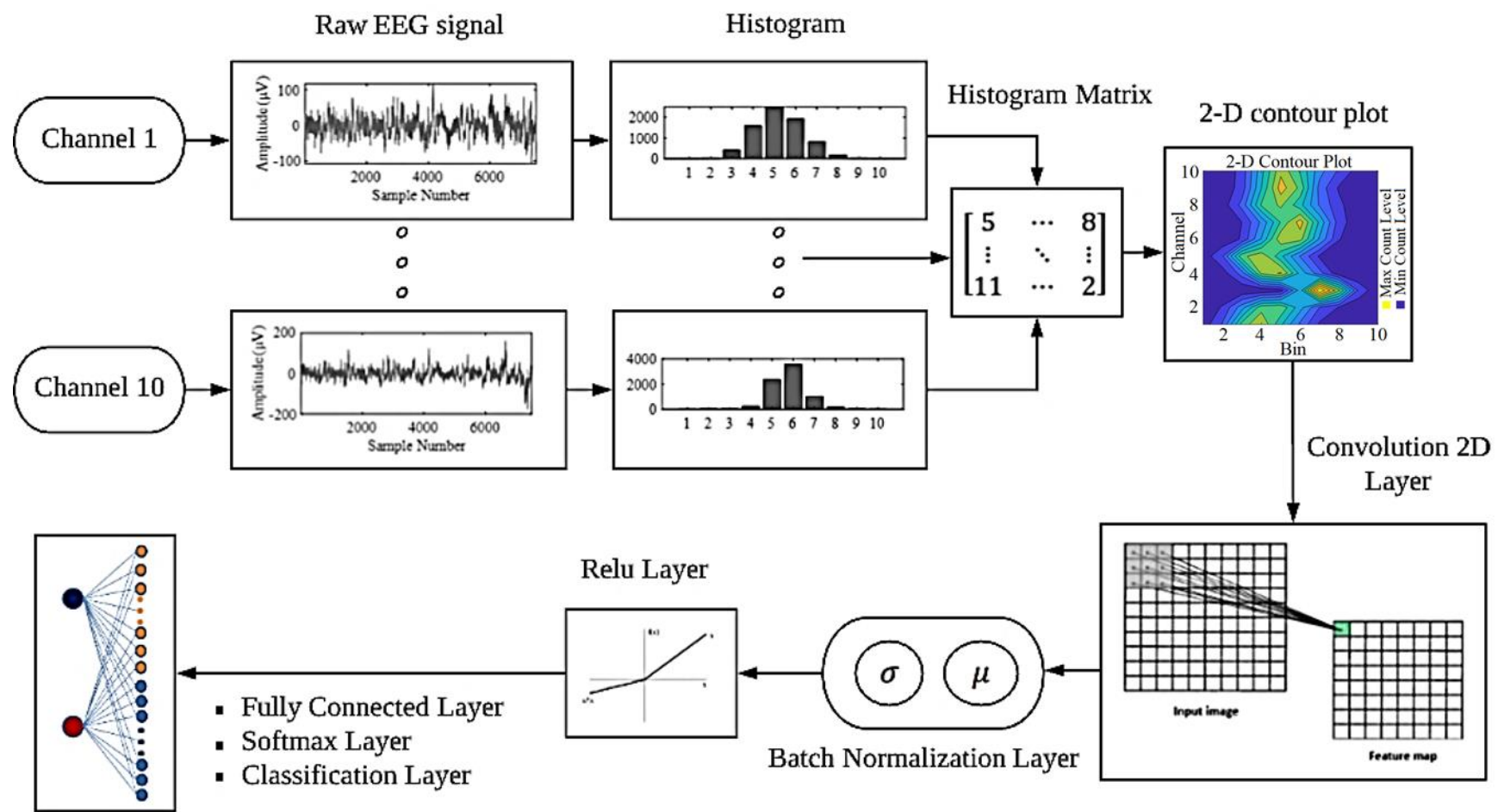

Figure 2. Architecture of proposed method

TABLE II. PERFORMANCE OF DEEP LEARNING MODEL OVER OBSERVATIONS IN THE MINI-BATCH

\begin{tabular}{|c|c|c|c|c|c|c|c|}
\hline Epoch & Iteration & $\begin{array}{c}\text { Time Elapsed } \\
\text { (hh:mm:ss) }\end{array}$ & $\begin{array}{c}\text { Mini-batch } \\
\text { Accuracy }\end{array}$ & $\begin{array}{c}\text { Validation } \\
\text { Accuracy }\end{array}$ & $\begin{array}{c}\text { Mini-batch } \\
\text { Loss }\end{array}$ & $\begin{array}{c}\text { Validation } \\
\text { Loss }\end{array}$ & $\begin{array}{c}\text { Base Learning } \\
\text { Rate }\end{array}$ \\
\hline 1 & 1 & $00: 00: 27$ & $34.38 \%$ & $0.00 \%$ & 0.9463 & 15.8386 & 0.0010 \\
\hline 3 & 50 & $00: 03: 01$ & $92.19 \%$ & $73.52 \%$ & 0.8425 & 3.7368 & 0.0010 \\
\hline 5 & 100 & $00: 05: 34$ & $100.00 \%$ & $97.02 \%$ & $2.9053 \mathrm{e}-05$ & 0.3151 & 0.0010 \\
\hline 7 & 150 & $00: 08: 09$ & $100.00 \%$ & $98.21 \%$ & $2.4028 \mathrm{e}-07$ & 0.1687 & $5.0000 \mathrm{e}-05$ \\
\hline 8 & 192 & $00: 10: 21$ & $100.00 \%$ & $96.37 \%$ & $7.4506 \mathrm{e}-09$ & 0.3922 & $5.0000 \mathrm{e}-05$ \\
\hline
\end{tabular}



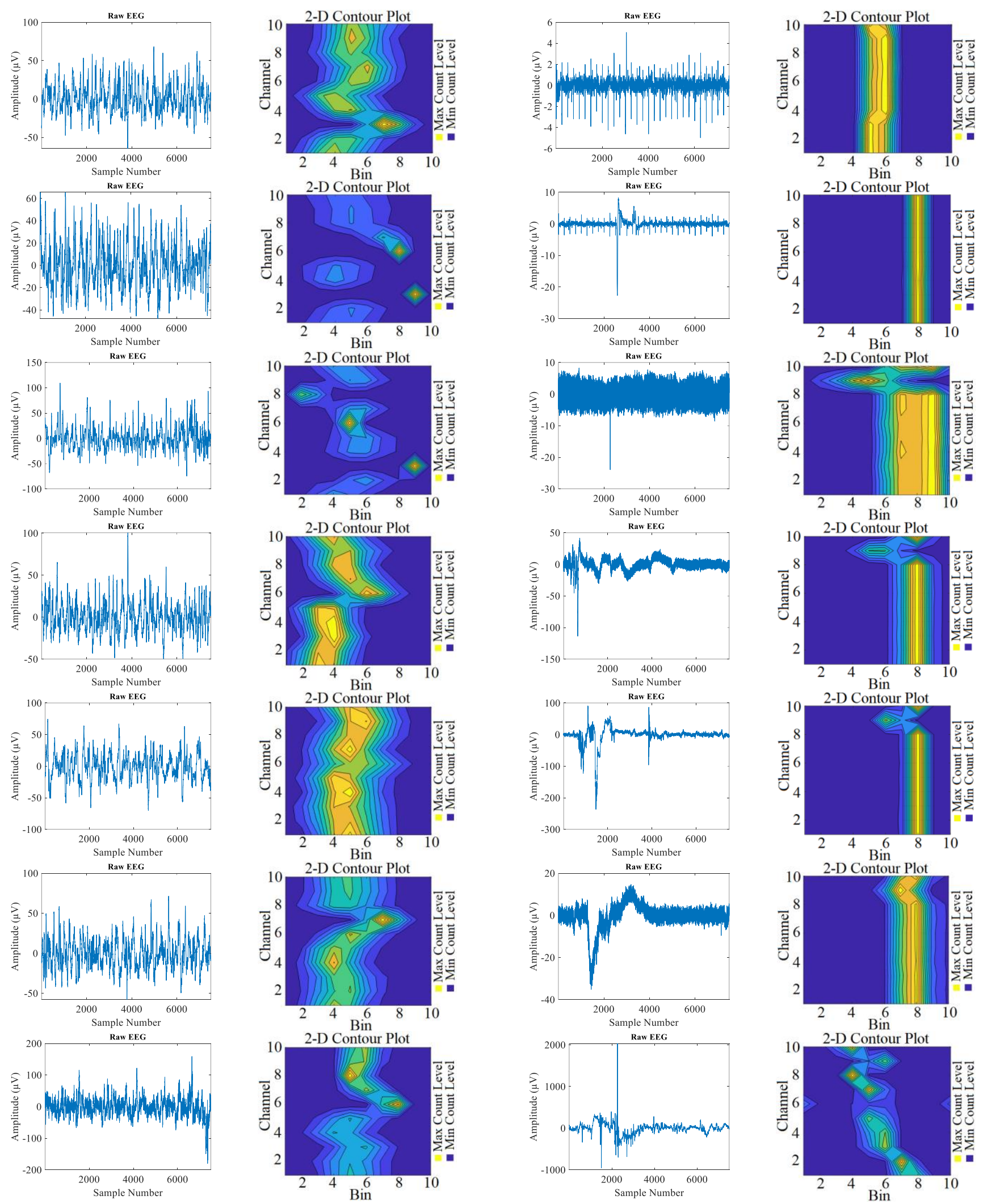

Figure 3.1. Raw EEG sequence

Figure 3.2. Histogram contour of all channels
Figure 4.1. Raw EEG sequence

Figure 4. Examples of EEG sequences including artifacts

Figure 3. Examples of artifact-free EEG sequences 


\section{RESULTS}

Table 2 summarizes the performance of deep learning model over the observations in the mini-batch. Figure 5 and 6 show the convergence of average accuracy and loss function during training and validation for 8 epochs. According to these figures, accuracy and loss improve for both training and validation data and the best result is obtained with validation accuracy of $98.21 \%$ and validation loss of 0.1687 .

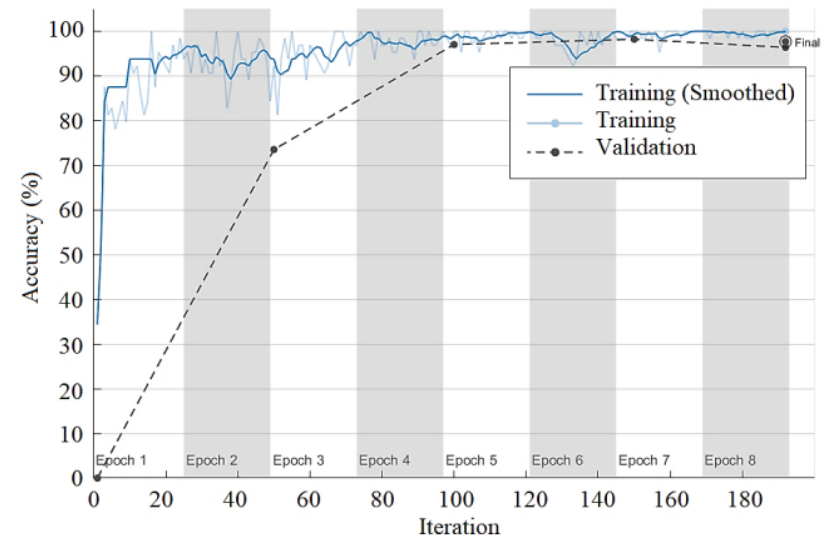

Figure 5. Accuracy variation in each epoch

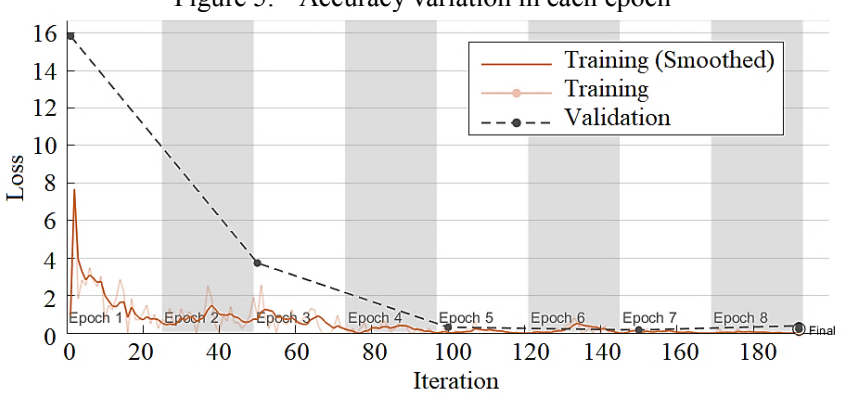

Figure 6. Loss function variation in each epoch

\section{CONCLUSION AND DISCUSSION}

This study introduced a simple and accurate method combining deep learning and histogram contour processing for automatic detection of artifacts in EEG recordings without requirement of auxiliary reference signal. Using the histogram contour was an extremely simple yet useful way of summarizing and representing what simultaneously happens in all EEG channels and therefore not only could be used to detect different types of artifact with different characteristics in both amplitude and frequency, but also takes into account spatial correlation of different EEG channels. The method was shown to be suitable in detecting artifacts from recordings collected with ICU-suitable electrode and device. The obtained results showed an overall accuracy of 0.98 , representing high reliability of proposed technique in detecting different types of artifacts and being comparable or outperforming the approaches proposed earlier in the literature reaching the accuracy of $67.59 \%$ for detecting four kinds of artifacts [18], and a median accuracy of $94.4 \%$ for classifying ocular and cardiac artifacts [19].

\section{REFERENCES}

[1] O. Dehzangi, M. Farooq: Portable Brain Computer Interface for the Intensive Care Unit Patient Communication using Subject-Dependent
SSVEP Identification, BioMed Research International, vol. 2018 , Article ID 9796238, 14 pages, (2018)

[2] E. Frank and et al.: Decreasing False Alarms by Obtaining the Best Signal and Minimizing Artifact from Physiological Sensors. Biomedical Instrumentation \& Technology: Nov./Dec. 2015, Vol. 49, No. 6, 423-431 (2015)

[3] M. Hravnak, L. Chen, A. Dubrawski, E. Bose, G. Clermont, M.R. Pinsky: Real alerts and artifact classification in archived multi-signal vital sign monitoring data: implications for mining big data. Journal of clinical monitoring and computing, 30 (6), 875-888 (2015)

[4] JH. Matsumoto, DL. McArthur, CW. Szeliga, JT. Lerner, LM. Rao, SA. Hussain, JY. Wu, MR. Nuwer, R. Sankar: Conductive plastic electrodes reduce EEG artifact during pediatric ECMO therapy. Journal of Clinical Neurophysiology, Official Publication of the American Electroencephalographic Society (2016)

[5] J. T. Valderrama, A. de la Torre, B. Van Dun: An automatic algorithm for blink-artifact suppression based on iterative template matching: Application to single channel recording of cortical auditory evoked potentials, J. Neural Eng., vol. 15, no. 1, 016008 (2018)

[6] H. Gonzalo Rey, C. Pedreira, and R. Quian Quiroga: Past, present and future of spike sorting techniques. In: Brain research bulletin 119, 106117 (2015)

[7] T. Brouns: Automated signal processing for extracellular neural recordings, Radboud University (2018)

[8] J. Jaeyoon Jun et al: Real-time spike sorting platform for high-density extracellular probes with ground truth validation and drift correction. In: bioRxiv, 101030 (2017)

[9] E. Bak $`$ stein et al.: Methods for automatic detection of artifacts in microelectrode recordings. In: Journal of neuroscience methods 290 , 39-51, (2017)

[10] B. Singh and H. Wagatsuma: A removal of eye movement and blink artifacts from EEG data using morphological component analysis, Comput. Math. Methods Med., vol. 2017, Jan. 2017, Art. no. 1861645 (2017).

[11] M. Chavez, F. Grosselin, Aurore Bussalb, Fabrizio De Vico Fallani, Xavier Navarro-Sune: Surrogate-based artifact removal from singlechannel EEG, IEEE Trans.Neural Syst.Rehab.Eng. 26, no.3, 540-550 (2018)

[12] J. Levitt, A. Nitenson, S. Koyama, L. Heijmans, J. Curry, JT. Ross, S. Kamerling, CY. Saab: Automated Detection of Electroencephalography Artifacts in Human, Rodent and Canine Subjects using Machine Learning, Journal of Neuroscience Methods (2018)

[13] P. Nejedly, J. Cimbalnik, P. Klimes et al.: Intracerebral EEG Artifact Identification Using Convolutional Neural Networks. Neuroinformatics. 1-10 (2018)

[14] S. ORegan, S. Faul, W. Marnane: Automatic detection of EEG artefacts arising from head movements using EEG and gyroscope signals. Medical engineering physics, 35 (7): 867-874 (2013)

[15] M. Mohammadpour and V. Rahmani, "A Hidden Markov Model-based approach to removing EEG artifact," 2017 5th Iranian Joint Congress on Fuzzy and Intelligent Systems (CFIS), Qazvin, pp. 46-49 (2017)

[16] S.H ORegan: Artifact detection and removal algorithms for EEG diagnostic systems (2013)

[17] Radüntz, T.; Scouten, J.; Hochmuth, O.; Meffert, B. Automated EEG artifact elimination by applying machine learning algorithms to ICAbased features. J. Neural Eng. 14, 046004 (2017)

[18] D. Kim and S. Keene, "Fast Automatic Artifact Annotator for EEG Signals Using Deep Learning" IEEE SPMB 2019, Pennsylvania, USA, (2019)

[19] A. Hasasneh, N. Kampel, P. Sripad, N. Jon Shah, and J. Dammers, "Deep Learning Approach for Automatic Classification of Ocular and Cardiac Artifacts in MEG Data," Journal of Engineering, vol. 2018, Article ID 1350692, 10 pages, (2018)

[20] P. Nejedly, J. Cimbalnik, P. Klimes et al. "Intracerebral EEG Artifact Identification Using Convolutional Neural Networks," Neuroinform 17: 225 (2019)

[21] J. Kortelainen, E. Väyrynen, and et. al, "Forehead electrodes sufficiently detect propofol-induced slow waves for the assessment of brain function after cardiac arrest," J Clin Monit Comput. (2019)

[22] S. Myllymaa and et. al. "Assessment of the suitability of using a forehead EEG electrode set and chin EMG electrodes for sleep staging in polysomnography.” J Sleep Res. 25(6): 636-45, (2016) 